\title{
Small Dense Low Density Lipoprotein and Cardiovascular Events in Patients With Acute Coronary Syndrome Undergoing Percutaneous Coronary Intervention: A Cohort Study From China
}

Chengping $\mathrm{Hu}$

Beijing anzhen hospital, Capital Medical University

Hongya Han

Beijing Anzhen Hospital, Capital Medical University

Yan Sun

Beijing anzhen hosital, capital medical university

\section{Yujing Cheng}

Beijing anzhen hospital, capital medical university

Yan Liu

Beijing anzhen hospital, capital medical university

Jinxing Liu

Beijing anzhen Hospital, Capital Medical University

\section{Ang Gao}

Beijing anzhen hospital,capital medical university

Yujie Zhou

Beijing anzhen hospital, capital medical university

Jianwei Zhang

Beijing anzhen hospital, capital medical university

Yingxin Zhao ( $\Delta$ zyingxinmi@163.com )

Beijing Anzhen Hospital, Capital Medical University https://orcid.org/0000-0002-4897-2779

\section{Original investigation}

Keywords: small dense LDL cholesterol, acute coronary syndrome, percutaneous coronary intervention, risk assessment

Posted Date: September 25th, 2020

DOl: https://doi.org/10.21203/rs.3.rs-81372/v1

License: (9) (1) This work is licensed under a Creative Commons Attribution 4.0 International License. Read Full License 


\section{Abstract}

Background: Residual risk remained significant despite effective low density lipoprotein cholesterol (LDL-C) lowering treatment. Small dense low density lipoprotein cholesterol (sdLDL-C) as part of LDL-C has been found to be predictor of coronary heart disease (CHD) and cardiovascular (CV) events in patients with stable CHD independently of LDL-C. However, to date, few studies have explored the role of sdLDL-C in patients with acute coronary syndromes (ACS) undergoing percutaneous coronary intervention (PCI). Accordingly, this study aimed to evaluate the association of sdLDL-C with CV events in patients with ACS undergoing PCI.

Methods: Patients hospitalized with ACS undergoing PCl were enrolled and followed up for 18 months. The risk of sdLDL-C for CV events was compared according to sdLDL-C quartiles. The primary outcome was the composite of death, nonfatal myocardial infarction, nonfatal stroke and unplanned repeat revascularization. A Cox proportional hazards regression model was performed to estimate the risk of CV events. Subgroup analysis according to diabetes status and dichotomized low-density lipoprotein cholesterol (LDL-C) and triglyceride (TG) level based on median value were performed separately for cardiovascular risk.

Results: A total of 6092 patients were included in the analysis (age: $60.2 \pm 10.13$ years, male: $75.3 \%$, BMl: $25.9 \pm 3.33 \mathrm{~kg} / \mathrm{m}^{2}$, dyslipidemia: $74.1 \%$ and diabetes: $\left.44.5 \%\right)$. During 18 months of follow-up, $320(5.2 \%)$ incident $\mathrm{CV}$ events occurred. Compared to the lowest sdLDL-C quartile group, patients in the highest quartile had a greater risk of $\mathrm{CV}$ events after multivariable adjustment (HR: 1.92; 95\% Cl: 1.37-2.70). In the subgroup analyses, this greater risk remained significant in patients, regardless of high or low LDL-C or TG (dichotomized by the median value) and diabetes status.

Conclusions: Patients with elevated sdLDL-C have a higher risk of CV events in Chinese patients with ACS undergoing $\mathrm{PCl}$, providing additional value for better risk assessment.

\section{Background}

Death rates related to cardiovascular disease (CVD) have decreased, but it was still a leading cause of deaths as a result of aging, obesity and diabetes mellitus (DM) $₫ 1 \rrbracket$. Dyslipidemia are widely recognized as a contributing risk factor for coronary heart disease (CHD) and stroke $2-3 \bigotimes$. However, residual risk remained significant despite effective low density lipoprotein cholesterol (LDL-C) lowering treatment in accordance with current guideline, including statin, ezetimibe and proprotein convertase subtilisin/kexin type 9 (PCSK9) antibodies $₫ 4-6 \rrbracket$. In addition, LDL-C comprised of a heterogeneous lipoprotein particles including large, more buoyant LDL particles (Ib-LDL) and small dense LDL (sdLDL) particles $87-8 \rrbracket$, which could change independently from LDL-C 8 9 . Compared with Ib-LDL, sdLDL-C had higher ability for penetration into arterial wall, lower binding affinity for receptor, longer plasma half-life and easier to oxidation $₫ 10 \otimes$. Due to the atherogenic properties of sdLDL-C, using LDL-C alone may underestimate actual risk in individuals when we evaluated cholesterol-related CHD risk $₫ 11$. Therefore, risk assessment may be benefit from sdLDL-C measurement.

sdLDL-C has been found to be associated with increased risk for the development of CHD among the healthy participants with high or low risk of CVD $₫ 12-13 \rrbracket$ and increased risk for cardiovascular (CV) events in patients with stable CVD 14 . However, to date, few studies have explored the role of sdLDL-C in patients with acute 
coronary syndromes (ACS) undergoing percutaneous coronary intervention (PCI). Accordingly, this study aimed to evaluate the association of sdLDL-C with CV events in patients with ACS undergoing PCI.

\section{Methods}

\section{Study Design and Patients}

In this cohort study, we consecutively included 9282 patients hospitalized for ACS and PCl from a top-ranked cardiovascular hospitals in China from January 2018 to December 2018. The main exclusion criteria were a body mass index $(\mathrm{BMI})>45 \mathrm{~kg} / \mathrm{m}^{2}$, left ventricular ejection fraction (LVEF) $<30 \%$, severe hepatic and renal insufficiency (eGFR<30 ml/min), suspected familial hypertriglyceridemia (triglyceride $\geq 5.65 \mathrm{mmol} / \mathrm{L}$ ), fibrate use, and malignancy diseases. The study protocol was approved by the institutional review board of Beijing Anzhen Hospital, Capital Medical University with a waiver of informed consent. Participants' personal details were concealed.

\section{Measurements}

The data including patient demographics, smoking status, past medical history, laboratory results, $\mathrm{PCl}$ data, and medical treatments were collected from medical and nursing records. Blood samples were drawn after an overnight at least 8 hours fasting. For patients with STEMI, blood samples were collected immediately on admission. Lipid profile were measured on the same day of collection. Fasting plasma glucose (FPG), glycosylated hemoglobin (HbA1c), Total cholesterol (TC) and triglyceride (TG) were measured by standard laboratory techniques. The measurement of sdLDL-C was performed in an automated homogeneous assay (Denka Seiken Co., Ltd., Tokyo, Japan) and analyzed on a Hitachi 7180 automatic analyzer》15区.

\section{Treatment and Procedure}

All medication and operation were performed according to the guidelines $₫ 16 \bigotimes$. All patients received aspirin and clopidogrel or ticagrelor prior to the procedure and $70-100 \mathrm{IU} / \mathrm{kg}$ unfractionated heparin intraoperative. A radial approach was used by 6 or $7 \mathrm{~F}$ guiding catheters. Second-generation drug eluting stents was implanted following appropriate predilation. The type of stent, fractional flow reserve (FFR), intravascular ultrasound (IVUS) and optical coherence tomography (OCT) were at the discretion of the interventionalist.

\section{Outcomes}

All patients were followed up to incident primary outcome or for 18 months by telephone and only index events were included in the analysis. All events were recorded by two telephone records and inconsistent events were affirmed by a third record. Hospital records were also screened for clinical events. The primary outcome was the composite of death, nonfatal MI, nonfatal stroke or unplanned repeat revascularization. Death was defined as all causes of death regardless of cause of death 17 . Myocardial infarction was defined as the criteria for the fourth universal definition $\nabla 18 \bigotimes$. Stroke was adjudicated by the presence of as acute cerebral infarction established by the imaging or typical symptoms $₫ 19 \bigotimes$. Unplanned repeat revascularization

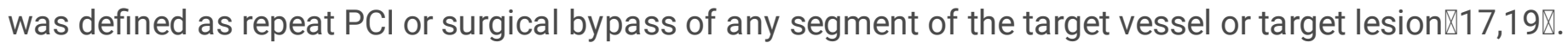
Unstable angina was defined as rest, new-onset, or worsening angina without cardiac enzyme elevation $\varangle 20 \rrbracket$. 
Obesity was defined as BMI $\geq 28 \mathrm{~kg} / \mathrm{cm}^{2}$. Hypertension was defined as a systolic blood pressure $\geq 140 \mathrm{~mm}$ $\mathrm{Hg}$, diastolic blood pressure $\geq 90 \mathrm{~mm} \mathrm{Hg}$, or use of antihypertensive medications $₫ 21 \rrbracket$. Diabetes mellitus was defined as taking hypoglycemic agents, a fasting ( $\geq 8$ hours) blood glucose of $\geq 7.0 \mathrm{mmol} / \mathrm{L}$, or a nonfasting

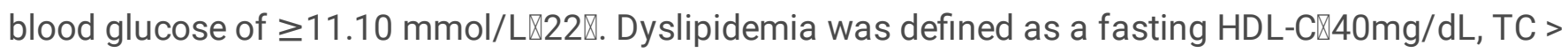
$200 \mathrm{mg} / \mathrm{dL}, \mathrm{LDL}-\mathrm{C}>130 \mathrm{mg} / \mathrm{dL}, \mathrm{TG}>150 \mathrm{mg} / \mathrm{dL}$ or use of any lipid-lowering drug.

\section{Statistical Analyses}

Baseline patient characteristics were presented according to baseline sdLDL-C quartiles. Continuous variables were expressed as the mean \pm standard deviation (SD) or median (interquartile range). The differences were estimated by one-way Analysis of Variance (ANOVA) for normal data or Kruskal-Wallis tests for non-normal distribution data followed by Bonferroni's post hoc test. Categorical variables are expressed as numbers (percentage) and compared with a $\chi 2$ test or Fisher's exact test.

Survival analyses were performed using Kaplan-Meier methods, log rank tests, and Cox proportional hazards regression models with backward stepwise selection methods according to baseline sdLDL-C quartiles. The following three models were adjusted for multivariate analysis: Model 1: age, gender, BMl; Model 2: model 1 + smoking status, hypertension, previous Ml, previous stroke, syntax score, number of stents, total length of stents. Model 3: model 2 + HDL-C, high sensitivity C-reactive protein (hs-CRP), lipid-lowering medication use. Additionally, subgroups stratified according to diabetes status and dichotomized LDL-C and TG level (based on median value) were analyzed separately for cardiovascular events. Subgroup analyses were also conducted in each subgroup of age, sex, obesity, hypertension, previous MI, ACS type, hs-CRP. The heterogeneities in the relationship between subgroups were evaluated by adding multiplicative interaction terms in the multivariable models. In addition, we present the comparisons of baseline characteristics between participants who were eligible or not for the final analyses to test whether missing data would potential bias the results. All statistical analyses were performed using SPPS 24.0 software (IBM Corp., Armonk, NY, USA). A two-tailed value of $\mathrm{P}<0.05$ was required for statistical significance.

\section{Results}

There were 9282 patients who met the inclusion criteria, of whom 3190 were excluded due to loss to follow-up $(n=781)$ or meet the major exclusion criteria $(n=2409)$. Finally, a total of 6092 patients were included in the analysis. Additional file 1: Figure S1 shows the patients' flowchart. Comparison of baseline characteristics between participants who were eligible or not for the final analyses was displayed in Additional file 1: Table S1. Compared with the lost participants, eligible participants were significantly older. Though statistically significant, differences in BMI, current smoker and hypertension were not clinically relevant. Additionally, there was no statistically significant difference in lipid parameters.

\section{Baseline Characteristics}

The sdLDL-C had an approximately normal distribution with a mean of $28.2 \pm 13.16 \mathrm{mg} / \mathrm{dl}$ (Additional file 1: Figure S2). Among high or low LDL-C (defined by the median of LDL-C) group of patients, wide variation in sdLDL-C was observed (Additional file 1: Figure S3a-b). With respect to diabetes status, significant differences of sdLDL-C were found ( $p=0.002$, Additional file 1: Figure S3c-d). Baseline characteristics data presented in 
Table 1. Among the included patients, there were 4586 (75.3\%) male and mean \pm SD age was $60.2 \pm 10.13$ years and BMI $25.9 \pm 3.33 \mathrm{~kg} / \mathrm{m} 2$. Diabetes and dyslipidemia was seen in $44.5 \%(2712)$ and $74.1 \%$ (4512) of patients, respectively. For the ACS type, $86.8 \%$ were unstable angina and the others were acute myocardial infarction (AMI). Among 2712 subjects with diabetes, 2171 were treated with oral hypoglycemic agents or insulin. Overall, almost all of patients were taking at least one prescription lipid-lowering medication and $98.4 \%$ were taking a statin with or without ezetimibe (20\%). Of the analyzed coronary artery lesions, $16.9 \%$ were in left main artery, 59\% were multivessel lesions, $15.2 \%$ were CTO lesion and the mean syntax score was 14 \pm 7.49 . FFR, IVUS and OCT were not widely practiced. The comparison of baseline characteristics according to sdLDL-C quartile are also shown in Table 1(Quartile 1:囚18.5mg/dL, Quartile 2:18.5-25.5 mg/dL, Quartile 3: 25.5-35.1 mg/dL, Quartile 4: $\geq 35.1 \mathrm{mg} / \mathrm{dL}$ ). There was statistically significant variation in age, gender, obesity, current smoking, hypertension, diabetes, dyslipidemia, hs-CRP and LVEF lipid parameters.

\section{Relationship sdLDL-C of with cardiovascular events}

A total of 320 (5.2\%) incident cardiovascular events occurred during 18 months of follow-up. Hazard ratios for incidence of CV events by quartile of sdLDL-C are presented in Table 2. Patients are grouped in sdLDL-C quartiles, with Quartile 1 having the lowest and Quartile 4 the highest risk of CV events after adjusted with model 3 (HR: 1.92; 95\% Cl: 1.37-2.70). In subgroup analyses according to diabetes status (Fig.1), the difference of HR between the extreme quartile of sdLDL-C remained statistically significant in model 3 (nondiabetes: HR 2.98, 95\% Cl 1.28-6.95; diabetes: HR 2.30, 95\% Cl 1.45-3.67). And there was no statistically significant interaction between sdLDL-C and diabetes status ( $P$ for interaction=0.789). Fig.2 exhibits the effect of sdLDL-C in combination with other lipid parameters on CV events. Using group of patients with sdLDL-C区 $25.48 \mathrm{mg} / \mathrm{dl}$ and LDL-C $₫ 86.85 \mathrm{md} / \mathrm{dl}$ as a reference, patients in the high sdLDL-C had a greater risk of CV events after adjusted with model 3 (Fig.2a, HR:1.62,95\%Cl 1.25-2.09, $P$ for interaction=0.728). Moreover, patients with high sdLDL-C and high LDL-C had the highest risk of CV events in model 3 (HR:1.92, 95\% Cl 1.372.70). In the stratified analyses by dichotomized TG level, similar pattern of results for adjusted risk for $\mathrm{CV}$ events were found (Fig.2b, $P$ for interaction=0.158).

Finally, stratified analysis by age, sex, obesity, hypertension, previous MI, ACS type, hs-CRP was conducted as shown in Fig.3. The multivariable-adjusted risk for CV events tended to be higher in subjects with highest quartile of sdLDL-C than in those with lowest quartile of sdLDL-C with or without statistically significant in all of subgroups analyzed in model 3. No significant interaction between sdLDL-C and these subgroups was

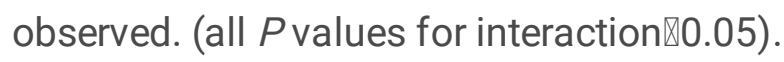

\section{Discussion}

In this study, we report that patients with a high sdLDL-C were more likely to be have a high risk of CV events in Chinese patients with ACS undergoing $\mathrm{PCl}$. This higher risk remained significant in patients regardless of diabetes status, LDL-C and TG levels. To our knowledge, our study is the first large-scale trial estimating the association between sdLDL-C and the risk of CV events in patients with ACS undergoing PCl. Actually, there

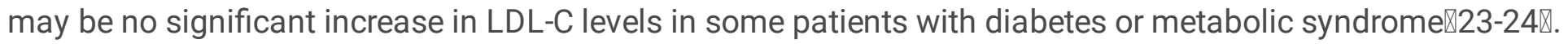
Therefore, it is clinically valuable to measure sdLDL-C for estimating the risk of CV events in patients with ACS 
undergoing PCl for its highly atherogenic properties. Overall, sd-LDL-C was favorable for distinguishing patients with high risk of CV events beyond LDL-C level.

There are several observational studies reported the associations between sdLDL-C and subclinical atherosclerosis $₫ 13,25-26 \rrbracket$, or CAD $111-12,27 \rrbracket$. In a small prospective study, an increase of sdLDL-C was shown to predict intima media thickness (IMT) and insulin resistance $\mathbb{2} 28$. Results from a trial with 816 patients without diabetes or CVD showed SdLDL-C can independently predict arterial stiffness progression $₫ 29 \rrbracket$. Moreover, several studies have indicated that sdLDL-C was independently associated with the progression of carotid atherosclerosis $₫ 13,25-26 \rrbracket$. The Suita Study followed 2,034 general urban Japanese residents for an average of 11 years and have suggested that the highest quartile of sdLDL-C level was associsated with a 3.3fold higher risk of incident CHD compared with the lowest quartile $(95 \% \mathrm{Cl}, 1.3-8.2) \varangle 30 \rrbracket$. The Multi-Ethnic Study of Atherosclerosis, which included 4387 USA patients and followed up for an average of 8.5 year, demonstrated that adjusted hazard ratios for incident CHD between extreme quartile of sdLDL-C was 2.4冈11》. The ARIC study, which included 11,419 patients and followed up for 11-year, demonstrated that sdLDL-C was associated with incident CHD®31囚. And the association remained significant regardless of LDL-C levels in these studies. Meanwhile, Duran EK et al. also indicated that sdLDL-C affected atherogenesis independently of LDL-C and hs-CRP $₫ 32 \rrbracket$, which was consistent with results of the above mentioned research. Furthermore, sdLDL-C predicted the CHD risk not only in patients at high cardiovascular risk $₫ 12 \bigotimes$, but also at low cardiovascular risk according to LDL-C values $₫ 31 \bigotimes$, therefore providing additional value for better risk assessment.

In addition, several studies reported the association between sdLDL-C and coronary stenosis severity or prognosis in patients with CAD. Koba $S$ et al. recruited 482 stable CHD patients and 389 patients without CHD and indicated sdLDL-C level was more efficacious in predicting coronary severity $₫ 33 \bigotimes$. A cohort study from china suggested that increased sdLDL-C were associated with higher risk of CV events in patients with diabetes and stable $C A D \otimes 14 \rrbracket$. Therefore, the current study might provide valuable further information on the relationship of sdLDL-C and CV events in patients with ACS undergoing PCl.

Also, several studies have shown sdLDL-C was closely related to stroke $26 \rrbracket$. A cross-sectional study included a total of 754 acute ischemic stroke (AIS) patients indicated that sdLDL-C levels was an independent predictor of NIHSS scores and the severity of cerebral artery calcification $₫ 34 \rrbracket$. A study enrolled 530 elderly patients hospitalized within $48 \mathrm{~h}$ after stroke suggested high sdLDL-C were associated with a greater risk for ischemic stroke $₫ 35 \rrbracket$. Another study recruited 355 AIS and 171 non-AIS patients and found that elevated sdLDL-C was associated with a higher incidence of AIS $₫ 36$.

In this study, we also report that sdLDL-C was associations with increased risk of CV events regardless of diabetes status, which seems to be not very consistent with previous study. The Multi-Ethnic Study of Atherosclerosis demonstrated that elevated sdLDL-C was an independent risk factors for CHD only in non-

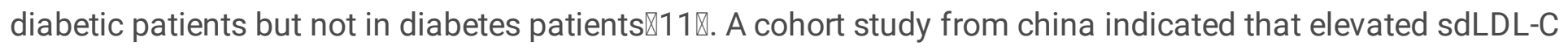
was associated with greater risk of $\mathrm{CV}$ events in DM patients with stable CAD but not non-diabetic patients

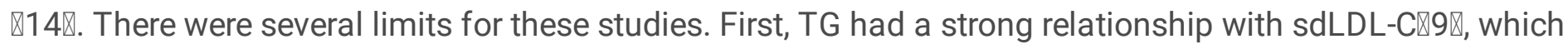
means the inclusion of both variables in the final multivariable regression model may bias the results. Second, the small sample size in the subgroup may explain the overall positive results but negative in subgroup 
analysis in these studies. Therefore, the negative result should not be used as a definite conclusion. Actually diabetic and non-diabetic patients accounted for almost half of the patients in the final analysis in our study, which means that our research may come up with positive results. In another study, the relationship between

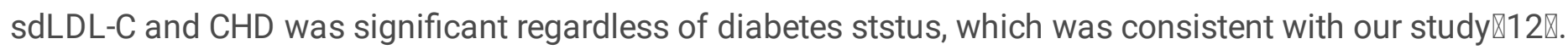
Moreover, patients with DM was likely to have smaller LDL囚37囚. sdLDL-C had strong association with metabolic syndrome $₫ 8 \rrbracket$, insulin resistance $₫ 9,39 \rrbracket a n d$ subclinical diabetes status $₫ 40 \rrbracket$.

The multiple characteristics of sdLDL-C, including greater propensity for endothelial penetration, lower affinity with LDL receptors, longer time in circulation and greater susceptibility to desialylation, glycation and oxidation, played an important role in the atherosclerosis $₫ 31 \rrbracket$. Krychtiuk et al. showed that sdLDL-C was associated with an increase of non-classical monocytes (NCM; CD14+CD16++) and a decrease of classical monocytes (CM; CD14++CD16-) $\llbracket 41 \rrbracket$. In a prospective study within the Women's Health Study, sdLDL-C was a strong risk factor for MI but not peripheral artery disease (PAD), which indicated that elevated sdLDL-C may be relevant to instability and vulnerability plaque rather than the more stable plaque $₫ 32 \rrbracket$. Compared with LDL-C, sdLDL-C are more vulnerable to oxidative and easily engulfed by macrophages $₫ 42-43 \rrbracket$, which strongly

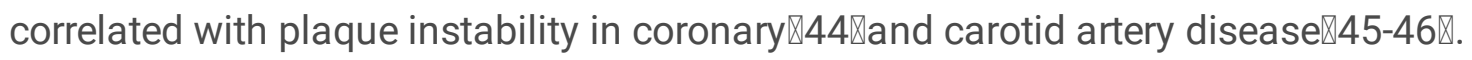

This study has several limitations to consider. First, our results may be affected by residual confounding in this observational study. Second, there may be a significant decrease in sdDL-C in patients with AMI, but our study still found a close relationship between preoperative sdLDL-C concentration and prognosis. In addition, we collected blood samples immediate after admission to reducing the effect on sdLDL-C. Third, timedependent analysis was not available for only once measurement of sdLDL-C at baseline. Fourth, unable to obtain follow-up information of medical treatments may bias the results. Finally, current research findings may not be generalizable to other ethnic groups because the participants in our study were only Chinese. Therefore, our findings should be confirmed in other ethnic populations.

\section{Conclusions}

Among Chinese patients with ACS undergoing PCl, patients with high sdLDL-C were at a higher risk of developing $\mathrm{CV}$. These findings may help identify high-risk patients with cardiovascular events beyond LDL-C and those patients may benefit from more aggressive therapy.

\section{Abbreviations}

$\mathrm{BMI}$, body mass index; FPG, fasting plasma glucose; HbA1C, Glycosylated hemoglobin; TC, total cholesterol; HDL-C, high-density lipoprotein-cholesterol; LDL-C, low-density lipoprotein-cholesterol; sdLDL-C, small dense low-density lipoprotein cholesterol; ACS, acute coronary syndrome; AMI, acute myocardial infarction; ACEI, angiotensin converting enzyme inhibitor; $\mathrm{ARB}$, angiotensin II receptor blocker; $\mathrm{PCl}$, percutaneous coronary intervention; CTO, chronic total occlusion; LM, left main artery, LAD, left anterior descending artery; LCX, left circumflex artery; RCA, right coronary artery; FFR, Fractional Flow Reserve; IVUS, intravascular ultrasound; OCT, optical coherence tomography; $\mathrm{HR}$, hazard ratio; $\mathrm{Cl}$, confidence interval.

\section{Declarations}


Acknowledgements

Not applicable.

\section{Authors' contributions}

Yingxin Zhao, Chengping Hu and Jianwei Zhang contributed to the conception and design of this study and Chengping Hu wrote this article. All authors made contribution to collect and analyze data. All authors read and approved the final manuscript.

\section{Funding}

This work was supported by the grant from National Key Research and Development Program of China (2017YFC0908800) Beijing Municipal Administration of Hospitals' Ascent Plan (DFL20150601) and Mission plan (SML20180601) Beijing Municipal Health Commission "Project of Science and Technology Innovation Center" (PXM2019_026272_000006) (PXM2019_026272_000005)

\section{Availability of data and materials}

The datasets used during the current study are available from the corresponding author on reasonable request.

\section{Ethics approval and consent to participate}

The study protocol was approved by the institutional review board of Beijing Anzhen Hospital, Capital Medical University with a waiver of informed consent. Information related to the identity of the patient was concealed.

\section{Consent for publication}

Not applicable.

\section{Competing interests}

The authors declare no competing interests.

\section{References}

1. Sidney S, Quesenberry CP, Jaffe MG, Sorel M, Nguyen-Huynh MN, Kushi LH, et al. Recent trends in cardiovascular mortality in the United States and public health goals. JAMA Cardiol. 2016; 1:594-9.

2. Prabhakaran D, Jeemon P, Roy A. Cardiovascular diseases in India: current epidemiology and future directions. Circulation. 2016;133: 1605-1620

3. HerringtonW, Lacey B, Sherliker P, Armitage J, Lewington S. Epidemiology of atherosclerosis and the potential to reduce the global burden of atherothrombotic disease. Circ Res. 2016;118: 535-546

4. Cholesterol Treatment Trialists' (CTT) Collaboration, Baigent C, Blackwell L, Emberson J, Holland LE, Reith $C$, et al. Efficacy and safety of more intensive lowering of LDL cholesterol: a meta-analysis of data from 170,000 participants in 26 randomized trials. Lancet. 2010; 376:1670-81. 
5. Cannon CP, Blazing MA, Giugliano RP, McCagg A, White JA, Theroux P, et al. Ezetimibe added to statin therapy after acute coronary syndromes. N Engl J Med. 2015;372: 2387-97.

6. Sabatine MS, Giugliano RP, Keech AC, Honarpour N, Wiviott SD, Murphy SA, et al. Evolocumab and clinical outcomes in patients with cardiovascular disease. N Engl J Med. 2017; 376:1713-22.

7. Superko HR, Gadesam RR. Is it LDL particle size or number that correlates with risk for cardiovascular disease? Curr Atheroscler Rep. 2008;10: 377-385.

8. Zhao X, Sun D, Xu RX, Guo YL, Zhu CG, Wu NQ, et al. Low-density lipoprotein-associated variables and the severity of coronary artery disease: an untreated Chinese cohort study. Biomarkers. 2018;23:647-653

9. Santos HO, Earnest CP, Tinsley GM, Izidoro LFM, Macedo RCO. Small dense low-density lipoproteincholesterol (sdLDL-C): Analysis, effects on cardiovascular endpoints and dietary strategies. Prog Cardiovasc Dis. 2020; undefined: undefined.

10. Kaneva AM, Potolitsyna NN, Bojko ER. Usefulness of the LDL-C/apoB ratio in the overall evaluation of atherogenicity of lipid profile. Arch Physiol Biochem. 2017;123:16-22

11. Tsai MY, Steffen BT, Guan W, McClelland RL, Warnick R, McConnell J, et al. New automated assay of small dense low-density lipoprotein cholesterol identifies risk of coronary heart disease: the Multi-ethnic Study of Atherosclerosis. Arterioscler Thromb Vasc Biol. 2014;34:196-201

12. Higashioka M, Sakata S, Honda T, Hata J, Yoshida D, Hirakawa Y, et al. Small dense low-density lipoprotein- 1 cholesterol and the risk of coronary heart disease in a Japanese community. J Atheroscler Thromb. 2020: 27:669-682

13. Qi Y, Liu J, Wang W, Wang M, Zhao F, Sun J, et al. High sdLDL Cholesterol can be Used to Reclassify Individuals with Low Cardiovascular Risk for Early Intervention: Findings from the Chinese MultiProvincial Cohort Study. J Atheroscler Thromb. 2020; 27: 695-710.

14. Jin JL, Zhang HW, Cao YX, Liu HH, Hua Q, Li YF, et al. Association of small dense low-density lipoprotein with cardiovascular outcome in patients with coronary artery disease and diabetes: a prospective, observational cohort study. Cardiovasc Diabetol. 2020; 19: 45

15. Ito $Y$, Fujimura M, Ohta M, Hirano T. Development of a homogeneous assay for measurement of small dense Idl cholesterol. Clin Chem. 2011; 57: 57-65

16. Neumann FJ, Sousa-Uva M, Ahlsson A, Alfonso F, Banning AP, Benedetto U, et al. 2018 ESC/EACTS Guidelines on myocardial revascularization. Eur Heart J. 2019; 40: 87-165.

17. Cutlip DE, Windecker S, Mehran R, Boam A, Cohen DJ, van Es GA, et al. Clinical End Points in Coronary Stent Trials. A Case for Standardized Definitions. Circulation. 2007; 115: 2344-2351.

18. Thygesen K, Alpert JS, Jaffe AS, Chaitman BR, Bax JJ, Morrow DA, et al. Fourth Universal Definition of Myocardial Infarction. Circulation. 2018;138: e618-e651.

19. Hicks KA, Tcheng JE, Bozkurt B, Chaitman BR, Cutlip DE, Farb A, et al. 2014 ACC/AHA Key Data Elements and Definitions for Cardiovascular Endpoint Events in Clinical Trials: A Report of the American College of Cardiology/American Heart Association Task Force on Clinical Data Standards (Writing Committee to Develop Cardiovascular Endpoints Data Standards). Circulation. 2015; 132: 302-61.

20. Roffi M, Patrono C, Collet JP, Mueller C, Valgimigli M, Andreotti F, et al. 2015 ESC Guidelines for the management of acute coronary syndromes in patients presenting without persistent ST-segment 
elevation: Task Force for the Management of Acute Coronary Syndromes in Patients Presenting without Persistent ST-Segment Elevation of the European Society of Cardiology (ESC). Eur Heart J. 2016; 37: 267315.

21. Williams B, Mancia G, Spiering W, Agabiti Rosei E, Azizi M, Burnier M, et al. 2018 ESC/ESH Guidelines for the management of arterial hypertension. Eur Heart J. 2018;39: 3021-3104.

22. Cosentino F, Grant PJ, Aboyans V, Bailey CJ, Ceriello A, Delgado V, et al. 2019 ESC Guidelines on diabetes, pre-diabetes, and cardiovascular diseases developed in collaboration with the EASD. Eur Heart J. 2020; 41: 255-323.

23. Hsu H, Hsu P, Cheng MH, Ito Y, Kanda E, Schaefer EJ, et al. Lipoprotein Subfractions and Glucose Homeostasis in Prediabetes and Diabetes in Taiwan. J Atheroscler Thromb. 2019; 26: 890-914

24. Sniderman A, Vu H, Cianflone K. Effect of moderate hypertriglyceridemia on the relation of plasma total and LDL apo B levels. Atherosclerosis. 1991;89:109-16

25. Norata GD, Raselli S, Grigore L, Garlaschelli K, Vianello D, Bertocco S, et al. Small dense LDL and VLDL predict common carotid artery IMT and elicit an inflammatory response in peripheral blood mononuclear and endothelial cells. Atherosclerosis. 2009;206:556-62.

26. Shen $H, X u L$, Lu J, Hao T, Ma C, Yang H, et al. Correlation between small dense low-density lipoprotein cholesterol and carotid artery intima-media thickness in a healthy Chinese population. Lipids Health Dis. 2015;14:137

27. Ai M, Otokozawa S, Asztalos BF, Ito Y, Nakajima K, White CC, et al. Small dense LDL cholesterol and coronary heart disease: Results from the Framingham Offspring Study. Clin Chem. 2010; 56: 967-976

28. Gerber PA, Thalhammer C, Schmied C, Spring S, Amann-Vesti B, Spinas GA, et al. Small, dense LDL particles predict changes in intima media thickness and insulin resistance in men with type 2 diabetes and prediabetes-a prospective cohort study. PLoS ONE. 2013; 8: e72763

29. Li G, Wu HK, Wu XW, Cao Z, Tu YC, Ma Y, et al. Small dense low density lipoprotein-cholesterol and cholesterol ratios to predict arterial stiffness progression in normotensive subjects over a 5-year period. Lipids Health Dis. 2018; 17:27

30. Arai H, Kokubo Y, Watanabe M, Sawamura T, Ito Y, Minagawa A, et al. Small dense lowdensity lipoproteins cholesterol can predict incident cardiovascular disease in an urban Japanese cohort: The Suita Study. J Atheroscler Thromb. 2013; 20: 195-203

31. Hoogeveen RC, Gaubatz JW, Sun W, Dodge RC, Crosby JR, Jiang J, et al. Small dense low-density lipoprotein cholesterol concentrations predict risk for coronary heart disease: the atherosclerosis risk in communities (ARIC) study. Arterioscler Thromb Vasc Biol. 2014;34: 1069-1077

32. Duran EK, Aday AW, Cook NR, Buring JE, Ridker PM, Pradhan AD. Triglyceride-Rich Lipoprotein Cholesterol, Small Dense LDL Cholesterol, and Incident Cardiovascular Disease. J Am Coll Cardiol. 2020; 75: 21222135

33. Koba S, Yokota Y, Hirano T, Ito Y, Ban Y, Tsunoda F, et al. Small LDL-cholesterol is superior to LDLcholesterol for determining severe coronary atherosclerosis. J Atheroscler Thromb. 2008;15: 250-60.

34. Yao T, Long Q, Li J, Li G, Ding Y, Cui Q, et al. Small dense low-density lipoprotein cholesterol is strongly associated with NIHSS score and intracranial arterial calcification in acute ischemic stroke subjects. Sci 
Rep. 2020; 10: 7645.

35. Manabe Y, Morihara R, Matsuzono K, Nakano Y, Takahashi Y, Narai H, et al. Estimation of the presence of small dense lipoprotein cholesterol in acute ischemic stroke. Neurol Int. 2015; 7:5973

36. Zhou P, Liu J, Wang L, Feng W, Cao Z, Wang P, et al. Association of Small Dense Low-Density Lipoprotein Cholesterol with Stroke Risk, Severity and Prognosis. J Atheroscler Thromb. 2020; undefined: undefined.

37. Tani S, Yagi T, Atsumi W, Kawauchi K, Matsuo R, Hirayama A. Relation between low-density lipoprotein cholesterol/apolipoprotein $B$ ratio and triglyceride-rich lipoproteins in patients with coronary artery disease and type 2 diabetes mellitus: a cross-sectional study. Cardiovasc Diabetol. 2017; 16: 123

38. Nozue T, Michishita I, Ishibashi Y, Ito S, Iwaki T, Mizuguchi I, et al. Small dense low-density lipoprotein cholesterol is a useful marker of metabolic syndrome in patients with coronary artery disease. J Atheroscler Thromb. 2007; 14: 202-7.

39. Lee JE, Min SH, Lee DH, Oh TJ, Kim KM, Moon JH, et al. Comprehensive assessment of lipoprotein subfraction profiles according to glucose metabolism status, and association with insulin resistance in subjects with early-stage impaired glucose metabolism. Int J Cardiol. 2016; 225: 327-331.

40. Hsu SH, Jang MH, Torng PL, Su TC. Positive Association Between Small Dense Low-Density Lipoprotein Cholesterol Concentration and Biomarkers of Inflammation, Thrombosis, and Prediabetes in Non-Diabetic Adults. J Atheroscler Thromb. 2019; 26: 624-635

41. Krychtiuk KA, Kastl SP, Pfaffenberger S, Lenz M, Hofbauer SL, Wonnerth A, et al. Association of small dense LDL serum levels and circulating monocyte subsets in stable coronary artery disease. PLoS ONE. 2015; 10: e0123367

42. Carmena R, Duriez P, Fruchart JC. Atherogenic lipoprotein particles in atherosclerosis. Circulation. 2004;109: III2-7.

43. Austin MA, Breslow JL, Hennekens CH, Buring JE, Willett WC, Krauss RM. Low-density lipoprotein subclass patterns and risk of myocardial infarction. JAMA. 1988; 260:1917-21.

44. Ehara S, Ueda M, Naruko T, Haze K, Matsuo T, Ogami M, et al. Pathophysiological role of oxidized lowdensity lipoprotein in plaque instability in coronary artery diseases. J Diabetes Complications. 2002; $16: 60-4$.

45. Nishi K, Itabe H, Uno M, Kitazato KT, Horiguchi H, Shinno K, et al. Oxidized LDL in carotid plaques and plasma associates with plaque instability. Arterioscler Thromb Vasc Biol. 2002;22: 1649-54

46. Sigala F, Kotsinas A, Savari P, Filis K, Markantonis S, Iliodromitis EK, et al. Oxidized LDL in human carotid plaques is related to symptomatic carotid disease and lesion instability. J Vasc Surg. 2010;52:704-13

\section{Tables}

Table 1 Baseline characteristics of patients grouped by sdLDL-C quartile 


\begin{tabular}{|c|c|c|c|c|c|c|}
\hline sdLDL-C & Total & Quartile 1 & Quartile 2 & Quartile 3 & Quartile 4 & $\begin{array}{l}P \\
\text { value* }\end{array}$ \\
\hline & & $\nabla 18.5 \mathrm{mg} / \mathrm{dL}$ & $\begin{array}{l}18.5- \\
25.5 \mathrm{mg} / \mathrm{dL}\end{array}$ & $\begin{array}{l}25.5- \\
35.1 \mathrm{mg} / \mathrm{dL}\end{array}$ & $\geq 35.1 \mathrm{mg} / \mathrm{dL}$ & \\
\hline$N(\%)$ & 6092(100) & 1593(26.1) & $1479(24.3)$ & $1516(24.9)$ & 1504(24.7) & - \\
\hline Age, y & $60.2 \pm 10.13$ & $62.1 \pm 9.9$ & $60.7 \pm 9.97$ & $59.6 \pm 9.71$ & $58.2 \pm 10.54$ & ${ }^{0} .001$ \\
\hline Male,n (\%) & $4586(75.3)$ & $1229(77.2)$ & $1140(77.1)$ & $1143(75.4)$ & 1074(71.4) & 0.001 \\
\hline BMI,kg/m2 & $25.9 \pm 3.33$ & $25.2 \pm 3.27$ & $25.8 \pm 3.27$ & $26.3 \pm 3.38$ & $26.4 \pm 3.26$ & 0.001 \\
\hline Obesity,n(\%) & 1393(24.2) & $283(18.9)$ & $327(23.2)$ & $401(27.7)$ & $382(27.3)$ & $\begin{array}{l}0.001 \\
0\end{array}$ \\
\hline Heart rate,bpm & $72.1 \pm 12.02$ & $71.6 \pm 11.29$ & $71.9 \pm 12.12$ & $72.1 \pm 12.03$ & $73.1 \pm 12.59$ & 0.004 \\
\hline SBP, mmHg & $128.2 \pm 21.14$ & $126.9 \pm 20.8$ & $128.3 \pm 21.17$ & $128.1 \pm 21.22$ & $129.6 \pm 21.32$ & 0.005 \\
\hline \multicolumn{7}{|c|}{ Medical history and risk factors, $\mathrm{n}(\%)$} \\
\hline Current smoker & $2200(36.1)$ & $509(32)$ & $549(37.1)$ & $542(35.8)$ & $600(39.9)$ & ${ }^{\square} .001$ \\
\hline Hypertension & $3941(64.7)$ & $1029(64.6)$ & $914(61.8)$ & 1024(67.5) & $974(64.8)$ & 0.013 \\
\hline Diabetes & 2712(44.5) & 692(43.4) & $642(43.4)$ & $658(43.4)$ & $720(47.9)$ & 0.028 \\
\hline Dyslipidaemia & $4512(74.1)$ & 1123(70.5) & 1077(72.8) & 1118(73.7) & 1194(79.4) & 0.001 \\
\hline Previous MI & 723(11.9) & 199(12.5) & 191(12.9) & $190(12.5)$ & $143(9.5)$ & 0.013 \\
\hline Previous Stroke & $278(4.6)$ & $88(5.5)$ & $62(4.2)$ & $73(4.8)$ & $55(3.7)$ & 0.075 \\
\hline Previous PCI & 1453(23.9) & $386(24.2)$ & $388(26.2)$ & $358(23.6)$ & $321(21.3)$ & 0.019 \\
\hline Previous CABG & $153(2.5)$ & $48(3)$ & $36(2.4)$ & $37(2.4)$ & $32(2.1)$ & 0.456 \\
\hline \multicolumn{7}{|l|}{$\begin{array}{l}\text { Laboratory } \\
\text { Tests }\end{array}$} \\
\hline $\mathrm{Cr}, \mu \mathrm{mol} / \mathrm{L}$ & $77.9 \pm 51.62$ & $82 \pm 69.37$ & $77.1 \pm 46.53$ & $77.6 \pm 47.82$ & $74.7 \pm 35.16$ & 0.001 \\
\hline $\begin{array}{l}\text { eGFR, } \\
\mathrm{ml} / \mathrm{min} / 1.73 \mathrm{~m} 2\end{array}$ & $123.6 \pm 36.4$ & $121.3 \pm 32.87$ & $123.3 \pm 29.6$ & $123.4 \pm 29.68$ & $126.5 \pm 49.61$ & 0.001 \\
\hline $\mathrm{FBG}, \mathrm{mmol} / \mathrm{L}$ & $7 \pm 2.54$ & $6.8 \pm 2.32$ & $6.9 \pm 2.44$ & $7 \pm 2.42$ & $7.4 \pm 2.9$ & 0.001 \\
\hline HbA1C,\% & $6.6 \pm 1.38$ & $6.5 \pm 1.25$ & $6.6 \pm 1.3$ & $6.6 \pm 1.31$ & $6.8 \pm 1.6$ & ${ }_{0}^{0} 001$ \\
\hline $\mathrm{TC}, \mathrm{mmol} / \mathrm{L}$ & $158.8 \pm 41.14$ & $124.4 \pm 22.06$ & $145.1 \pm 25.08$ & $164 \pm 26.81$ & $203.5 \pm 39.17$ & $\begin{array}{l}\square \\
0.001\end{array}$ \\
\hline TG,mmol/L & 123.2(88.5- & 85.1(65.1- & 109.4(86.8- & $142.8(109.4-$ & 180.5(137.1- & ए \\
\hline
\end{tabular}




\begin{tabular}{|c|c|c|c|c|c|c|}
\hline & 176.2) & 107.6) & 145.8) & 189.2) & 243.9) & 0.001 \\
\hline HDL-C,mmol/L & $41.4 \pm 9.66$ & $42.2 \pm 10.16$ & $41.3 \pm 10.06$ & $40.5 \pm 9.44$ & $41.5 \pm 8.85$ & $\begin{array}{l}{[} \\
0.001\end{array}$ \\
\hline LDL-C,mmol/L & $93.2 \pm 34.31$ & $65 \pm 16.66$ & $83.2 \pm 20.12$ & $97.4 \pm 23.63$ & $128.8 \pm 35.87$ & $\begin{array}{l}\square \\
0.001\end{array}$ \\
\hline hs-CRP & $3.1 \pm 4.44$ & $2.7 \pm 4.3$ & $2.9 \pm 4.3$ & $3.1 \pm 4.27$ & $3.7 \pm 4.78$ & $\begin{array}{l}\square \\
0.001\end{array}$ \\
\hline TNI, $\mu g / L$ & $0.9 \pm 5.34$ & $0.7 \pm 4.87$ & $0.5 \pm 3.42$ & $0.9 \pm 5.46$ & $1.5 \pm 6.99$ & $\begin{array}{l}\square \\
0.001\end{array}$ \\
\hline LVEF,\% & $61.2 \pm 7.74$ & $60.8 \pm 7.65$ & $61 \pm 7.98$ & $61.4 \pm 7.61$ & $61.6 \pm 7.69$ & 0.302 \\
\hline \multicolumn{7}{|l|}{ ACS type, n (\%) } \\
\hline $\begin{array}{l}\text { Unstable } \\
\text { angina }\end{array}$ & $5286(86.8)$ & 1410(88.5) & 1303(88.1) & 1303(85.9) & $1270(84.4)$ & 0.002 \\
\hline AMI & $806(13.2)$ & 183(11.5) & 176(11.9) & $213(14.1)$ & 234(15.6) & - \\
\hline \multicolumn{7}{|c|}{ medication at discharge,n (\%) } \\
\hline Aspirin & $5956(97.8)$ & 1555(97.6) & 1451(98.1) & 1482(97.8) & 1468(97.6) & 0.770 \\
\hline clopidogrel & $4289(70.4)$ & $1130(70.9)$ & 1059(71.6) & 1027(67.7) & 1073(71.3) & 0.071 \\
\hline ticagrelor & 1968(32.3) & $504(31.6)$ & $469(31.7)$ & $530(35)$ & $465(30.9)$ & 0.079 \\
\hline ACEI/ARB & 2655(43.6) & $673(42.2)$ & $652(44.1)$ & $688(45.4)$ & $642(42.7)$ & 0.284 \\
\hline$\beta$-Blocker & $3926(64.4)$ & 1034(64.9) & $977(66.1)$ & $959(63.3)$ & $956(63.6)$ & 0.352 \\
\hline statin & $5996(98.4)$ & 1567(98.4) & 1455(98.4) & 1492(98.4) & 1482(98.5) & 0.981 \\
\hline Ezetimibe & $1219(20)$ & $259(16.3)$ & $255(17.2)$ & $331(21.8)$ & $374(24.9)$ & $\begin{array}{l}\square \\
0.001\end{array}$ \\
\hline $\begin{array}{l}\text { Any } \\
\text { antidiabetic } \\
\text { agents }\end{array}$ & 2171(35.6) & $562(35.3)$ & $509(34.4)$ & $516(34)$ & $584(38.8)$ & 0.024 \\
\hline \multicolumn{7}{|c|}{$\begin{array}{l}\text { Angiographic Coronary } \\
\text { anatomy, } \mathrm{n}(\%)\end{array}$} \\
\hline $\begin{array}{l}\text { Any left main } \\
\text { disease }\end{array}$ & 1029(16.9) & $262(16.4)$ & $223(15.1)$ & $273(18)$ & 271(18) & 0.094 \\
\hline $\begin{array}{l}\text { Multivessel } \\
\text { disease }\end{array}$ & $3597(59)$ & $885(55.6)$ & $818(55.3)$ & $916(60.4)$ & $978(65)$ & $\begin{array}{l}\square \\
0.001\end{array}$ \\
\hline Others & $2290(37.6)$ & $648(40.7)$ & $619(41.9)$ & $546(36)$ & $477(31.7)$ & $\begin{array}{l}\square \\
0.001\end{array}$ \\
\hline Сто & $928(15.2)$ & $233(14.6)$ & 253(17.1) & $218(14.4)$ & $224(14.9)$ & 0.141 \\
\hline $\begin{array}{l}\text { Lesions > } 20 \\
\mathrm{~mm}\end{array}$ & $3703(60.8)$ & $955(59.9)$ & $844(57.1)$ & $918(60.6)$ & $986(65.6)$ & $\begin{array}{l}{[]} \\
0.001\end{array}$ \\
\hline
\end{tabular}




\begin{tabular}{|lllllll|}
\hline $\begin{array}{l}\text { Syntax } \\
\begin{array}{l}\text { Treated vessel, } \\
\mathbf{n}(\%)\end{array}\end{array}$ & $14 \pm 7.49$ & $14.1 \pm 7.39$ & $13.5 \pm 6.97$ & $14.5 \pm 7.97$ & $14 \pm 7.55$ & 0.04 \\
\hline LM & $623(10.2)$ & $164(10.3)$ & $138(9.3)$ & $166(10.9)$ & $155(10.3)$ & 0.537 \\
\hline LAD & $3143(51.6)$ & $833(52.3)$ & $748(50.6)$ & $775(51.1)$ & $787(52.3)$ & 0.709 \\
\hline LCX & $1763(28.9)$ & $425(26.7)$ & $423(28.6)$ & $454(29.9)$ & $461(30.7)$ & 0.074 \\
\hline RCA & $2436(40)$ & $647(40.6)$ & $585(39.6)$ & $614(40.5)$ & $590(39.2)$ & 0.824 \\
\hline DCB & $375(6.2)$ & $110(6.9)$ & $83(5.6)$ & $89(5.9)$ & $93(6.2)$ & 0.471 \\
\hline FFR & $31(0.5)$ & $14(0.9)$ & $5(0.3)$ & $6(0.4)$ & $6(0.4)$ & 0.117 \\
\hline IVUS & $137(2.2)$ & $41(2.6)$ & $44(3)$ & $35(2.3)$ & $17(1.1)$ & 0.005 \\
\hline OCT & $108(1.8)$ & $31(1.9)$ & $23(1.6)$ & $27(1.8)$ & $27(1.8)$ & 0.877 \\
\hline $\begin{array}{l}\text { number of } \\
\text { stents }\end{array}$ & $1.7 \pm 0.82$ & $1.7 \pm 0.82$ & $1.6 \pm 0.8$ & $1.7 \pm 0.84$ & $1.7 \pm 0.82$ & 0.068 \\
\hline $\begin{array}{l}\text { Total length of } \\
\text { stents,mm }\end{array}$ & $39.9 \pm 24.5$ & $39.1 \pm 25.56$ & $38.8 \pm 23.24$ & $41.1 \pm 24.7$ & $40.4 \pm 24.52$ & 0.323 \\
\hline
\end{tabular}

Values are mean $\pm S D$, median (interquartile range), or $n(\%)$. * $p$ value for test of difference group of participants according to sdLDL-C quartile by one-way Analysis of Variance or $\chi 2$ test. BMI body mass index, SBP systolic blood pressure, MI myocardial infarction, $\mathrm{PCl}$ percutaneous coronary intervention, $\mathrm{CABG}$ Coronary Artery Bypass Grafting, $\mathrm{Cr}$ creatinine, UA Uric Acid, eGFR estimated glomerular filtration rate, FPG fasting plasma glucose, HbA1C Glycosylated haemoglobin, TC total cholesterol, TG triglyceride, HDL-C high-density lipoprotein-cholesterol, LDL-C low-density lipoprotein-cholesterol, sdLDL-C, Small dense low-density lipoprotein cholesterol, LVEF left ventricular ejection fraction, AMI acute myocardial infarction, ACEl angiotensin converting enzyme inhibitor, ARB angiotensin II receptor blocker, CTO chronic total occlusion, LM left-main artery, LAD left anterior descending artery, LCX left circumflex artery,RCA right coronary artery, DCB drug-coated balloon, FFR Fractional Flow Reserve, IVUS intravascular ultrasound, OCT optical coherence tomography

Table 2 Risk of cardiovascular events according to quartile of sdLDL-C

\begin{tabular}{|c|c|c|c|}
\hline & Model 1* & Model 2* & Model 3* \\
\hline Quartile 1 (reference) & - & - & - \\
\hline Quartile 2 & $1.31(0.91-1.89)$ & $1.28(0.88-1.87)$ & $0.73(0.45-1.19)$ \\
\hline Quartile 3 & $1.77(1.26-2.48)$ & $1.65(1.16-2.35)$ & $1.62(1.25-2.09)$ \\
\hline Quartile 4 & $2.33(1.68-3.23)$ & $2.21(1.57-3.09)$ & $1.92(1.37-2.70)$ \\
\hline$P$ for trend & 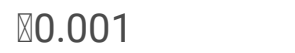 & $\otimes 0.001$ & 0.004 \\
\hline
\end{tabular}


Values are hazard ratio (95\% confidence interval) unless otherwise indicated. * Model 1: age, gender, BMl; Model 2: model 1 + smoking status, hypertension, previous MI, previous stroke, syntax score, number of stents, total length of stents. Model 3: model $2+$ HDL-C, high sensitivity C-reactive protein, lipid-lowering medication use. Hazard ratios and 95\% confidence intervals were obtained from Cox proportional hazards models.

\section{Figures}

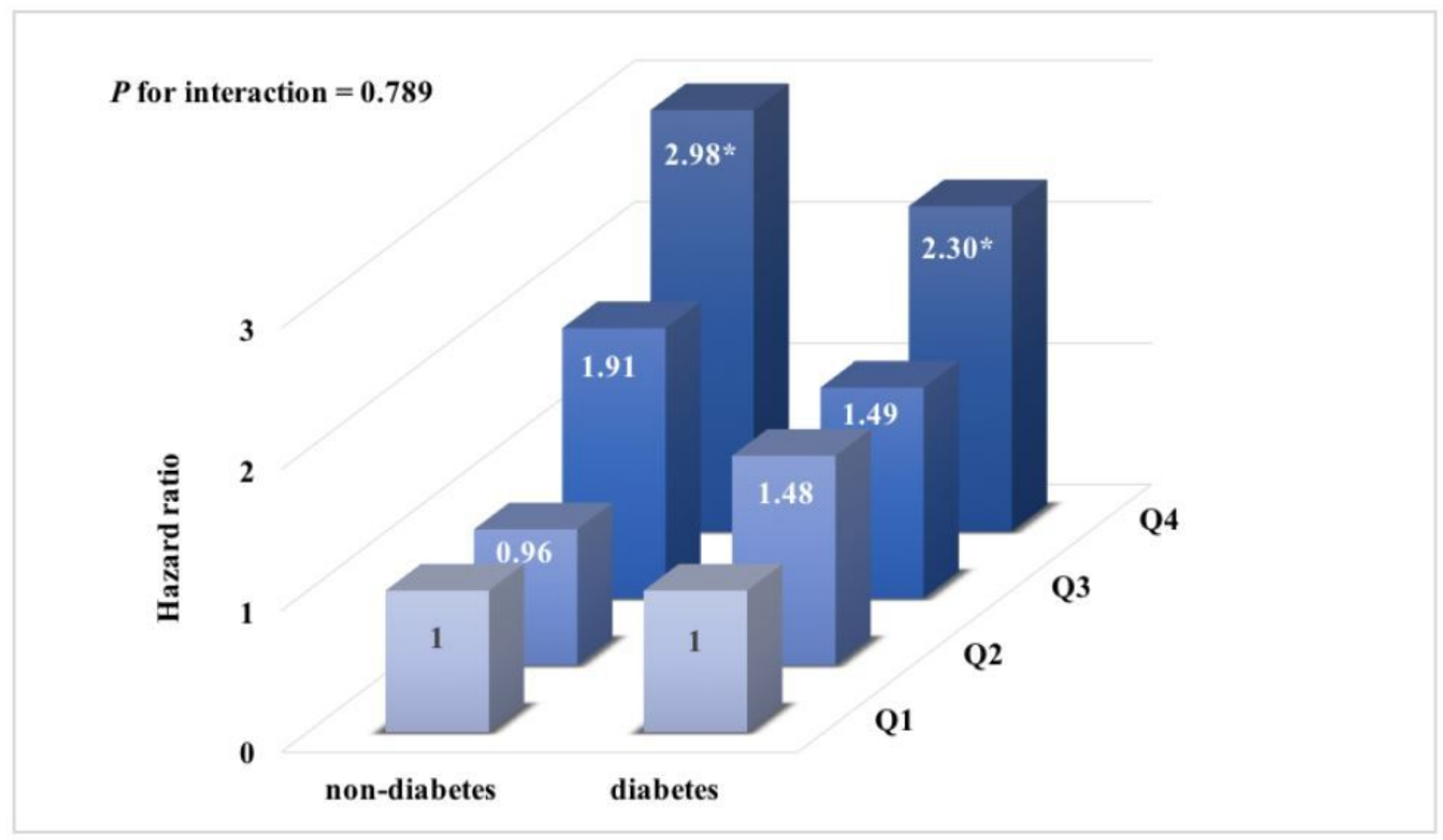

\section{Figure 1}

Hazard ratios for cardiovascular events according to sdLDL-C quartile and different diabetes status. sdLDL-C, small dense low-density lipoprotein cholesterol. *p囚0.05. Adjusted for age, gender, BMI, smoking status, hypertension, previous $\mathrm{MI}$, previous stroke, syntax score, number of stents, total length of stents, HDL-C, high sensitivity C-reactive protein, lipid-lowering medication use. 


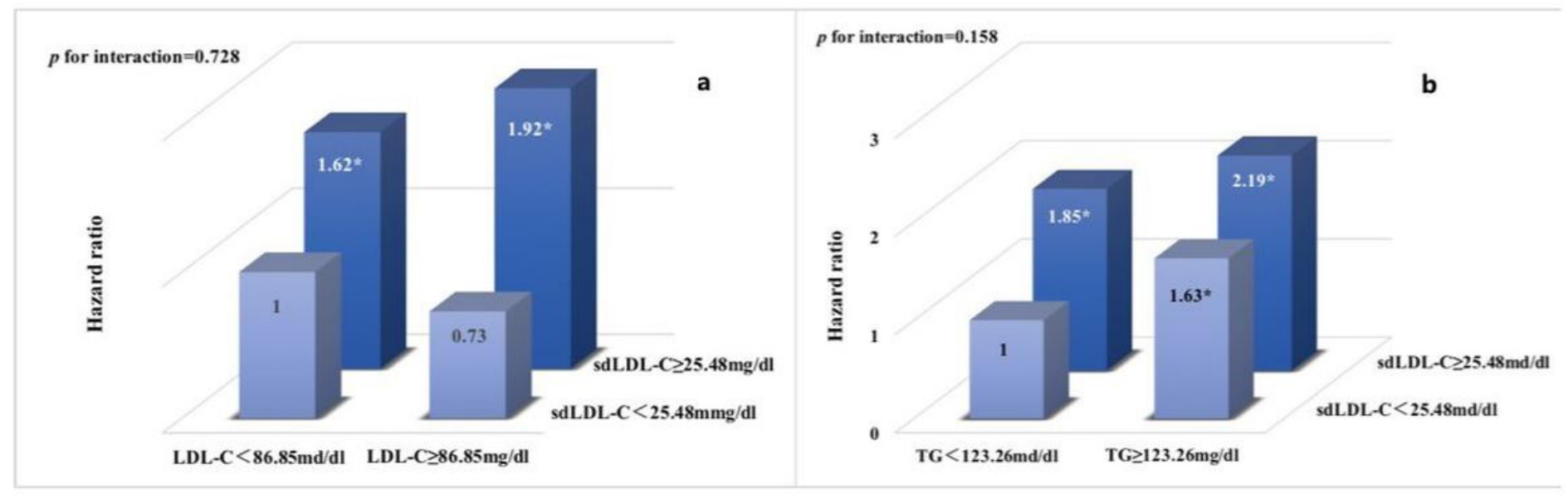

Figure 2

Hazard ratios for cardiovascular events according to sdLDL-C and different LDL-C and TG level. sdLDL-C, small dense low-density lipoprotein cholesterol. *p囚0.05. Adjusted for age, gender, BMI, smoking status, hypertension, previous MI, previous stroke, syntax score, number of stents, total length of stents, HDL-C, high sensitivity C-reactive protein, lipid-lowering medication use.

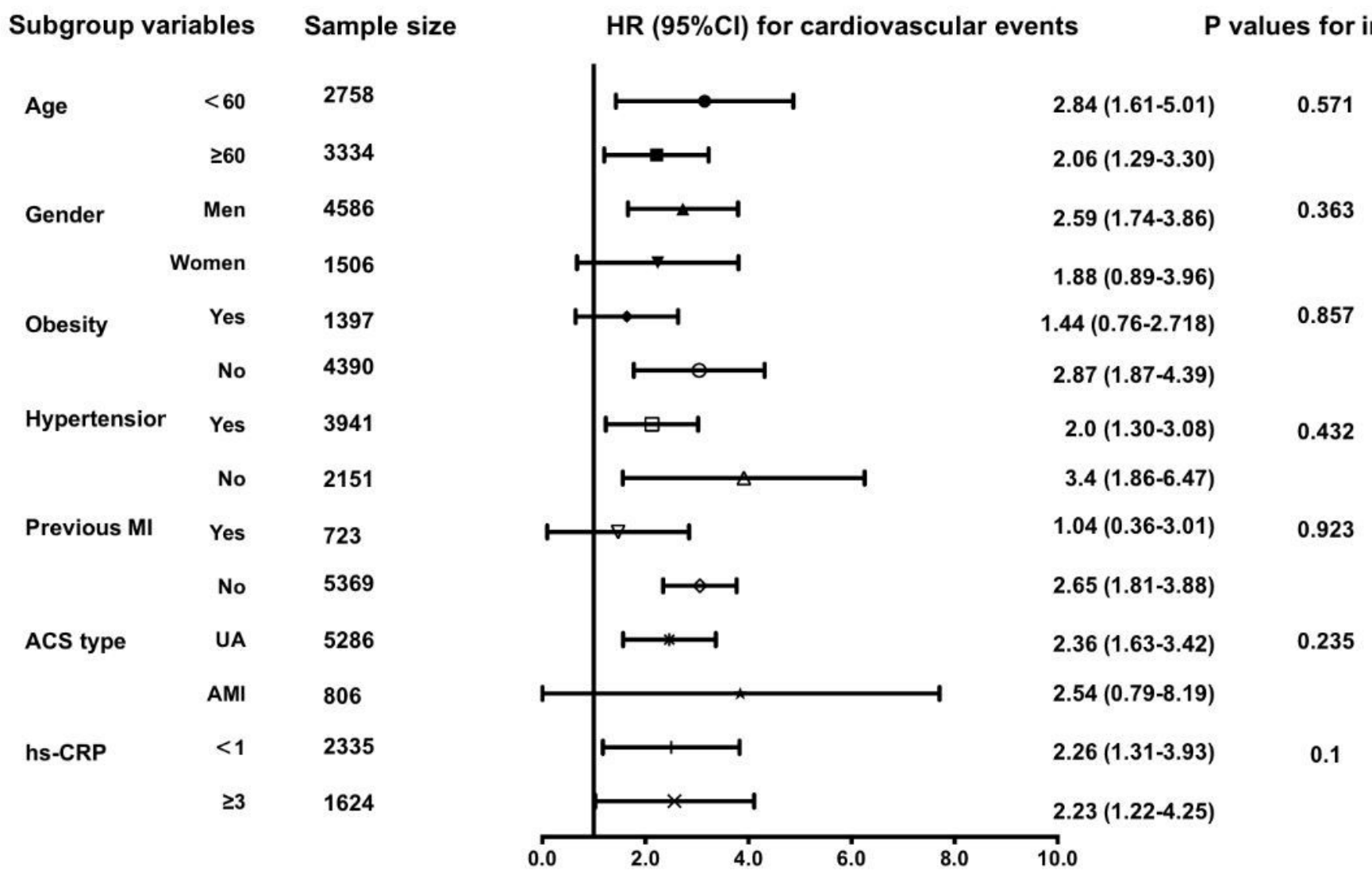

Figure 3 
Extreme quartile adjusted hazard ratios for associations with cardiovascular events in subgroups of cardiovascular risk factors. MI myocardial infarction, ACS acute coronary syndrome, sdLDL-C, Small dense low-density lipoprotein cholesterol. *p $₫ 0.05$. Adjusted for age, gender, BMI, smoking status, hypertension, previous $\mathrm{MI}$, previous stroke, syntax score, number of stents, total length of stents, HDL-C, high sensitivity Creactive protein, lipid-lowering medication use excluding subgroup variables

\section{Supplementary Files}

This is a list of supplementary files associated with this preprint. Click to download.

- supplement1.docx

- supplement2.doc 